ROTATION, VARIABILITY AND AGE OF Ap STARS

P. North

Institut d'Astronomie de l'Université de Lausanne

CH-1290 Chavannes-des-Bois

Switzerland

ABSTRACT. Photometrically determined gravities for field and cluster Ap stars, together with published periods, show that conservation of angular momentum alone is responsible for the observed increase of the periods with age. New photometric periods of a few cluster stars are presented which independently confirm this result. No outstanding change with age of the amplitude of lightcurves is noted.

1. RESULTS

\title{
1.1. Rotation
}

1.1.1. Ap stars in clusters. Table 1 lists a few periods of cluster stars recently obtained with the Geneva photometry. They confirm the already noticed lack of magnetic braking on the main sequence for CP2 and CP4 stars (Klochkova and Kopylov, 1984; North, 1984).

Table 1. New photometric periods of cluster Si stars

\begin{tabular}{|c|c|c|c|c|c|}
\hline HD/HDE & CLUSTER & PERIOD & $\begin{array}{l}\text { NO, OF } \\
\text { MEASURES }\end{array}$ & LOG AGE & AGE REF, \\
\hline 16605 & NGC 1039-37 & NOT VARIABLE & 19 & 8.3 & NoRth \& CRAMER 1981 \\
\hline 45583 & NGC $2232-9$ & $\begin{array}{rl}1,177 & \mathrm{D}, \\
\text { OR } & 6,60 \mathrm{D}\end{array}$ & 44 & 7.35 & MERMILLIOD 1980 \\
\hline 66295 & NGC $2516-26$ & 2.454 & 37 & 8.1 & \\
\hline 66318 & NGC 2516-24 & NOT VARIABLE & 38 & 8.1 & North \& Cramer 1981 \\
\hline 68074 & NGC $2547-5$ & $1.1696 \mathrm{D}$ & 21 & 7.65 & \\
\hline 145102 & UPPER SCO & $\begin{array}{l}1.42 \mathrm{D} . \\
(\mathrm{OR} 3.33 \mathrm{D} . ?)\end{array}$ & 38 & $\sim 7.0$ & KLOCHKOVA ET AL \\
\hline 304847 & NGC $3114-234$ & $\begin{array}{lll}2.3 & D_{1} ? \\
\text { OR } & 4.6 & 0 . ?\end{array}$ & 25 & 8.1 & NoRTh \& CRAmer 1981 \\
\hline
\end{tabular}

167

C. R. Cowley et al (eds.), Upper Main Sequence Stars with Anomalous Abundances, 167-170.

(C) 1986 by D. Reidel Publishing Company. 
References to Table 1

Klochkova, V.G., Kopylov, I.M., Kumaigorodskaya, R.N.: 1981, Sov. Astron. Lett. $7(3), 203$

Mermilliod, J.-C.: 1980, Thesis

North, P., Cramer, N.: 1981, in "Upper Main Sequence CP Stars", 23rd Liège Astrophys. Co11., p. 55

1.1.2 Field (and cluster) Ap stars with known gravity. The Geneva photometry gives a statistically reliable estimate of $10 g \mathrm{~g}$ for most Ap stars, except for a few exceptionally blanketed ones (North \& Cramer, 1984). Gravity is used as an age indicator in Fig. 1 and 2, where all stars with a known period (Catalano \& Renson, 1984) and good Geneva colours are plotted. The gravities are probably less reliable in Fig. 2 because of increased blanketing by metallic lines (Teff being smaller) and because of the sensitivity of the Balmer jump to peculiarity (Gerbaldi et al., 1974). A linear least-squares fit through all 92 points of Fig. 1 yields a correlation at the $99 \%$ confidence level $(r=0.387)$. Excluding the four anomalous points - whose initial period would be shorter than $0.5 \mathrm{~d}$, while so short periods have never been observed gives a better correlation $(r=0.553)$ and exactly the same slope $(b=-0.64)$ as that of the theoretical relation for the rather realistic case of rigid-body rotation (Endal \& Sofia, 1979). The much greater dispersion of the 89 points of Fig. 2 prevents any fit from being done, but the lower envelope is compatible with the theoretical line.

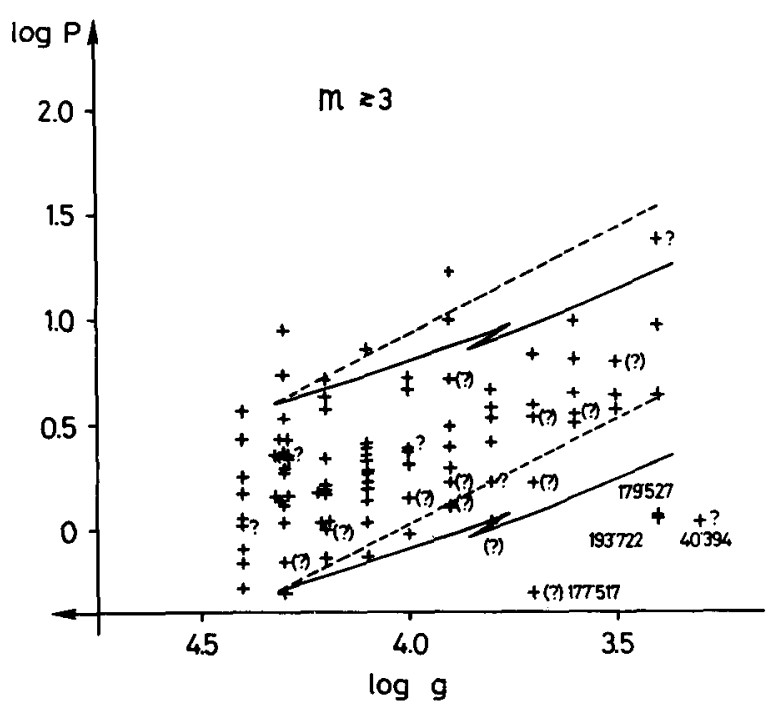

FIG. 1: PERIOD-GRAVITY RELATION FOR CP2 AND CP4 STARS MORE MASSIVE THAN $3 M_{0}$. CONTINUOUS LINES: THEORETICAL RELATIONS FOR A -4 MO STAR WITH COMPLETE RADIAL EXCHANGE OF ANGULAR MOMENTUM, WITH INITIAL PERIODS OF 0.5 AND 4.0 D.

BROKEN LINES: THEORETICAL RELATIONS IN THE CASE OF CONSERVATION OF ANGULAR MOMENTUM IN INDEPENDENT SHELLS. QUESTION MARKS ARE FOR UNCERTAIN OR AMBIGUOUS PERIODS. 


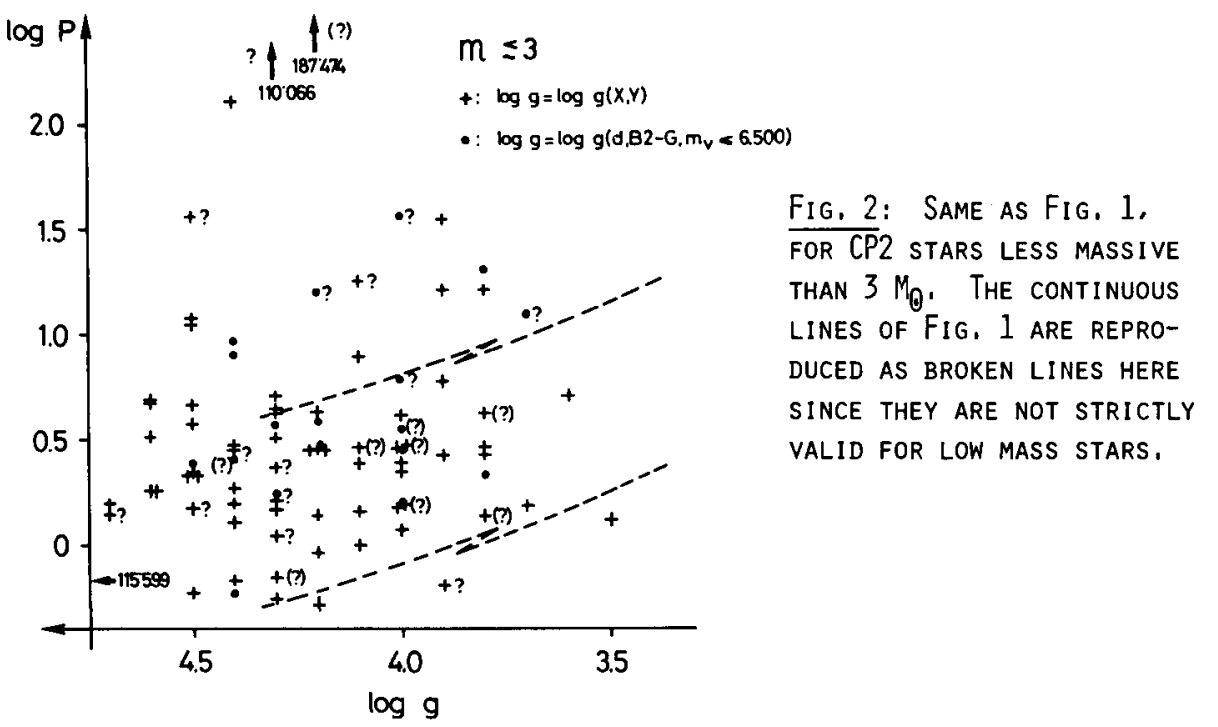

1.1.3 Initial distribution of periods. Neglecting the influence of the initial rotational velocity on its subsequent evolution, one may estimate the period each star had on the ZAMS from its present period and evolutionary state. The distribution of initial periods is shown in Fig. 3 for stars with $M>3 M_{\odot}$ and compared with the distribution for normal dwarfs. It is consistent with the observed periods of Ap stars in young associations.

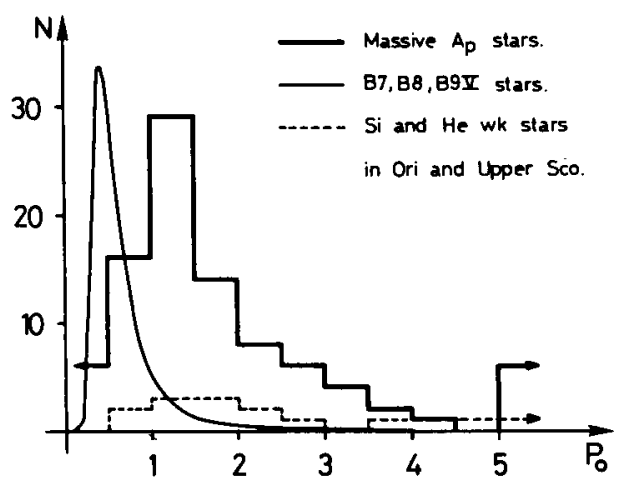

Fig. 3: Distribution of the

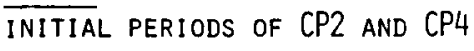
STARS WITH $M>3 M_{0}$. DEDUCED FROM FIG, 1. THE DISTRIBUTION OF NORMAL CLASS $V$ STARS IS SHOWN FOR COMPARISON,

\subsection{Lightcurve amplitudes}

Plots of the peak-to-peak amplitudes of an homogeneous sample of uvby lightcurves of 44 stars with any mass (Mathys \& Manfroid, 1985) vs $\log g$ shows no significant trend, except perhaps in the $u$ band, where less variation occurs at small gravities (Tables 2 to 5). More data are clearly needed. 
Tables 2 to 5 . Contingency tables for amplitudes in the u, v, $b$ and $y$ passbands. The $\chi^{2}$ value and corresponding level of significance at which the null hypothesis can be rejected are indicated and show no significant correlation

TABLE $2: U$

\begin{tabular}{c|c|c|c}
\hline LOG G & & & \\
\hline$>.06$ & 15 & 3 & 18 \\
\hline$\leqslant .06$ & 15 & 11 & 26 \\
\hline TOTAL & 30 & 14 & 44
\end{tabular}

$x^{2}=3.22, \quad-92 \%$

TABLE $4: B$

\begin{tabular}{c|c|c|c}
\hline$A(B)$ LOG G & $>3.9$ & $\leqslant 3.9$ & TOTAL \\
\hline$\geqslant .035$ & 17 & 6 & 23 \\
\hline$<.035$ & 13 & 8 & 21 \\
\hline TOTAL & 30 & 14 & 44
\end{tabular}

$$
x^{2}=0.73, \sim 60 \%
$$

TABLE $3: V$

\begin{tabular}{|c|c|c|c|}
\hline$A(v)$ & $>3.9$ & $\leqslant 3.9$ & TOTAL \\
\hline$>.03$ & 16 & 8 & 24 \\
\hline$<.03$ & 14 & 6 & 20 \\
\hline TOTA & 30 & 14 & 44 \\
\hline
\end{tabular}

$$
\begin{gathered}
x^{2}=0.06, \sim 20 \% \\
\text { TABLE } 5: y
\end{gathered}
$$

\begin{tabular}{c|c|c|c}
\hline A YOG G & $>3.9$ & $\leqslant 3.9$ & TOTAL \\
\hline$\geqslant .03$ & 16 & 5 & 21 \\
\hline$<.03$ & 14 & 9 & 23 \\
\hline TOTAL & 30 & 14 & 44
\end{tabular}

$$
x^{2}=1.19, \sim 70 \%
$$

\section{CONCLUSION}

No significant braking (magnetic or other) occurs during the MS phase of field as well as cluster magnetic Ap stars in the range 2.5 to $6 \mathrm{M}_{\odot}$. Conservation of angular momentum alone accounts for the change of period on the MS. Slow rotation of Ap stars is thus related to stellar formation.

\section{REFERENCES}

Catalano, F.A., Renson, P.: 1984, Astron. Astrophys. Supp1. 55, 371 Enda1, A.S., Sofia, S.: 1979, Astrophys. J. 232, 531

Gerbaldi, M., Hauck, B., Morguleff, N.: 1974, Astron. Astrophys. 30,105 Klochkova, V.G., Kopylov, I.M.: 1984, in "The Magnetic Stars", (eds.

V.L. Khokhlova, D.A. Ptitsyn, O.A. Lielausis), Salaspils, p. 78 Mathys, G., Manfroid, J.: 1985, Astron. Astrophys. Supp 1. 60, 17 North, P.: 1984, Astron. Astrophys. 141, 328 North, P., Cramer, N.: 1984, Astron. Astrophys. Supp 1. 58, 387

Discussion appears after the Musielok paper. 